labest one would have done so, I believe, even without artificial dilatation of the cervix. The placenta as shown to the above Society is a very interesting and typical specimen of placental apoplexy. There was no history of syphilis or tubercle in this case. On microscopic examination a section through the placenta at the edge of the infarcbshowed some disesse of the vessels, but no decided fatty change.

Leeds.

\section{GASTRO-JEJUNOSTOMY FOR PYLORIC CANCER; RECOVERY.}

BY F. A. PURCELL, M.D., M.CH., SURGEON TO THE CANCER HOSPITAL.

F. M - aged forty four, married and the mother of two children, was admitted in to the Cancer Hospital on March 3rd, 1892. The patient, a spare woman, weighing (nude) 5 st. $13 \frac{1}{4} \mathrm{lb}$, is suffering from cancer of the pylorus and stomach wall. She complains of pain in the stomach and the right hypochondrium; vomiting (no blood) comes on about two hours after taking food; bowels irregular, habitaally constipated, motions dark, with débris of blood; hearb has a reduplication of first sound; lungs sound; urine alkaline, contains no albumen. A distinct tumour is to be felt over the pylorus, nodulated and movable, extending from the right to across the middle line of the umbllicus. The stomach is enlarged; a succussion splash is distinctly produced. The nature of the disease was explained to the patient. The gradual wasting, constant vomiting, and libtle or no fæses passed, confirmed the serious outlook of gradnal starvation and exhanstion, caused by disease oscluding the outleb of the stumach. After a consultation at which the condition was confirmed by my colleagues, it was decided to explore the pylorus and, if feasible, to perform pylorectomy, and gastro jejunostomy by means of Senn's decalcified bone plates. The patient gave her ready consent to the resection of the pylorus. Preliminary to operabion, on March 11th, the stomach was commenced being daily washed out by means of a long soft rubber eyphon tube, passed into the stomach by raising the tube (wioh a funnel at top) and pouring the flaid in; then lowering the end below the bed, syphon action, the fluil was all drawn off, together with any débris of food and mucus; a quart of warm water was first used, then a quaro of a 2 per cent. solution of salicplate of soda, firishing with a quary of warm water to thoroughly get rid of the salicylate of soda. The passing of the tube and the washing out process gave no distress, unpleasantness, or discomfort to the patient. Natrient enemata were administered per rectum nighb and morning ; peptonised milk and essence of beef only were given by the mouth. This was continued for nine days. On March 18.h, the day prior to operation, the stomach was not washed out, owing to a feeling of weakness and faintness; that night the bowels were cleared out by a soapand-water injection. A $10 \mathrm{P} \mathrm{M}$. she partook of five onnces of peptonised milk.-March 19ih : Day of operating. Nothing given by the moutb. At 7 A M. a beef-bes enema was administered, and at 2 P.M., just prior to being placed on the table, an ounce of beef-tea and an ounce of brandy were administered per rectum. Full preparations were $m$ ide for resection of the pylorus, and for the gastro-jejunostomy two of Senn's deculcified bone plates were threaded with four long threads, the two end ones of Cainese silk, the two lateral of chromicised catgut; fine intestinal needles threaded with fine chromicised catgut for suturing the peritoneal surfaces together A hot-water bed was placed on the operating table for the patient to lie on to keep her warm. She was anæsthetised with chloroform by Dr. Bourns. I arranged with him, so soon as I had completed the incision in the abdominal wall and had thoroughly explored the diseased pylorus, that he should ease the patient out of the chloro form, supplying enough to simply control her. This Dr. Bourns satisfactorily carried out. Time occupied, fifty minutes; amount of chloroform used, six drachms; no sickness. I was assisted by my colleague, $\mathbf{M r}$. F. B. Jessett. A four.inch incision was made in the middle line from the umbilicus towards the ensiform cartilage. The stomash presented itself in a much enlarged condition, and the fice border on the right was implicated with disease for about four inches, extending into the pylorus and encircling the duodenum down to and fixing it to the spine below. Disease on the stomach wall was the movable tamour felt on examination. It was self-evident that the disease was too extensive to attempt resection of the pylorus. This, then, was abandoned. Gastro-jejunostomy was proceeded with. The jejunum was sought for and found deep down above the border of the left kidney and brought outside the abdominal wound. Its course towards the duodenum was travelled along until a part which came in easy apposition with the front wall of the stomach was selected to attach to the stomach. A piece about four inches was included between two round rubber elastic ligatures. These ligatures were lightly tied with one knot and clamped with torsion forceps to prevent slipping. The anterior wall of the stomach was drawn out and warm sponges packed around; a vertical fold was pinched up with the fingers, avoiding bloodvessels; a three-quarter inch incision was made with scissors through the entire coats ; the mucous membrane that protruded was pared off, and a few bleeding points were secured and bied. One of the bone plates was then inserted through the opening. The lateral threads of chromicised catgut armed with needles were passed through the entire coats from within outwards, about one-eighth of an inch from the edge of the wound. The end threads of silk were brought out at the ends of the opening, and not passed through the walls of the gut; the several threads were held so as not to crass or get mixed.

The portion of jejunum already isolated was now taken; its internal aspect was pinched ro into a transverse fold and cut across longitudinally three-quarters of an inch to correspond with the opening in the stomach; the mucous coat protruding was pared off, any bleeding vessel being tied; the second bone plate was passed in, and the several threads treated as above described. The threads were now drawn taut, and my assistant adapted the plates in apposition, the fingers of both hands encircling fixed them ; the bottom gut ligatures were now tied frmly, but not too much squeezed; the tying was done by drawing the threads longitudinally down between the surfaces; the corresp mnding end threads of silk were tied; and, lastly, the upper lateral ones of gut; ends of all were cut short. My assistant still keeping hold of the plates, I passed some six quilt ${ }^{1}$ sutures around the upper. border and ends, securing the peritoneal coats of the stomach and bowel together; the underborder wasnot touched. Theround rubber ligatures were released from the bowel. The toilet completed, sponging of the abdominal caviby was necessibated to geb rid of some ascitic flaid ; the channel seemed perfectly stanch. Theabdominal wound was brought together with silkworm gutligatures, dressings, and flunnel binder applied completed the operation, which lasted fifty minutes. Much time was wasted in exploring the pyloric disease. The patient was nice and warm, and came easily out of the chloroform, having consumed six drachms. I thank De. Bunrns for the way he managed the anrsthetic, and also thank my colleague Mr. Jessett for his kind assistance and for the admirable way he held the bone plates when inserted, which is a most material point in the operation.

That night, on account of severe pain, a hypodermic injection of one sixth of a grain of morphia was inserted in the arm, after which she slept for an hour and a half. She was given a teaspoonful of tepid water every half hour; no sickness; a few drops of brandy were added later on during the night; urine drawn (seven ounces) - 20th : Two teaspoonfuls of peptonised milk with the same quantity of water and four minims of brandy were given every hour; libtle pain. Slept three hours; urine drawn (nineteen ouncts). Brandy incressed to one ounce.-24th: Progressed since last report; sleep extended from six to seven hours. Milk-andwater has been partaken of freely, and to.day she has had mutton broth. Plenty of urine passed, no sickness, and little pain. -25 th: A soap-and-water enema relieved the bowels; dieb increased by addition of eggs; brandy discontinued.$26 \mathrm{~h}$ : Fish dinner, which she enjoyed. Bowels moved by simple enema; all sutures in abdominal incision were taken out. The wound looks well; the dressing had not been touched since first put on. - $27 \mathrm{bh}$ : Removed from special ward to the general ward. She soon made a move up to the conch, and began to partake of the usual meals, without any distress

1 The quilt or square suture for intestinal work was introduced by suture. 
or discomfort. Temperature on the $20 \mathrm{bh}$, at $10 \mathrm{~A}$.M., went up to $100^{\circ} \mathrm{F}$; ; on the $22 \mathrm{ad}$, at $10 \mathrm{~A}$. M., it was normal; it alightly varied until the $26 \mathrm{bh}$, af ter which it was not taken. Palse was steady all through, at 86 to 80 . The patient is improved in appearance, and has gained flesh. Discharged from hospital on April 14th.

I thank my house surgeon, Mr. Sylvester Willard, for the way he prepared the patient for operation, and the care he bestowed during the after treatment.

Manchester-square, $\mathbf{W}$.

\section{ACQUIRED DEAF-MUTISM.}

BY R. SHALDERS MILLER, M.B. LoND., F.R.C.S. ENG.

THE popular notion that the deaf and dumb are always born in that condition is by no means correct. Deafness mas be acquired in the early months of life, and if it persist io results in dumbness. I can offer no information as to the relative frequency of congenital and acquired cases of deaf-mutism, but I wish to show that in some instances, certainly, the acquired form may be remedied. Children often become deaf in the early years of life, and there seems to be no reason why the same should not occur in their early months; and in the latter event they will never learn to speak naturally unless the cause of deafness be removed. I have met with one example of the recovery of fair hearing without treatment, in adult life or in adolescence, in a man who had been regarded as a deaf-mate; but he never learned to converse, though he could utter a few short words, and could answer questions if they were so formulated as to allow of monosyllabic responses. This man had not learned "lip-speaking"; he heard ordinary sounds around him, but he always wrote his remarks if they needed to be put into several words. Another patient, a woman, presented a very similar history and condition, but was unable to articulate any intelligible sound; she replied to questions by signs only. She was, however, of somewhat feeble intelleco. The case to which I would more particularly call abtention is that of a child, F. P-, who was first brought to me in April, 1889, and was then four years old. She was absolutely deaf to all sounds around her, and it was impossible to get any trustworthy indication as to hearlng by conduction through the cranial bones. On examination the child was found to have slight otorrhœa, the membranes of the drums were somewhat retracted, there was chronic catarrh of the naso-pharynx, and the tonsils were greatly enlarged. I removed both tonsils, treated the meatuses and naso-pharynx by ordinary means, and inflated the tympana by Politzer's method. In a few days after treatment began the child gave clear indications of hearing, being startled by loud noises, and was evidently pleased with the sound of church bells \&e. She would also answer a knock ao the door, and imitated the "clucking" of poultry. For many weeks, however, she made no attempt to speak, and it was difficult for her parents and sister to overcome their constant habio of communicating with her by signs, for which she was extremely watchful. One of the earliest words the learno was her own name. In about six months she was sent to school with her sister, where she learnt much more quickly, especially from her school. fellows. She has had several relapses of deafness due to cold during the last three years, but has been promptly relieved by inflation. Her mother has laterly supplied me with a list of over sixty words which she pronounces plainly, one of the longest being "grandmother," and another (one of the most difficulo perhaps) "Irish." The list also contains some sentences, such as-" Wait a minute," "I'll tell fabher," \&c. She pronounces all the letters of the alphabet clearly except $G, K, N, Q, R, S$, and $\mathrm{Z}$, which are less distinctly articulated separately, though words containing those letters are fairly well spoken. She is not quick at figures, but names them perfectly up to seven, and less plainly up to twelve. Before her meals she says "grace," consisting of eleven words exclusive of "amen," which she calls "apen." She also repeats a prayer of nineteen different words, which are well articulated, though in a childlike fashion.

Another case of acquired (as I believe) deaf-mutism was that of a child two years old, who was brought to me for treatment about seven years ago. This child had never given evidence of possessing a trace of hearing. Her drums were retracted, but she had no perceptible catarrh of the external meatuses. I tried inflation, and at the first abtempt succeeded in filling the lefo tympanum with air, but not the right. After two or three visits the child noticed soundse g., she looked round sharply when I entered the room, though her back was towards the door. The first sound this patient gave evidence of hearing was the loud clacking noise of a metronome which I set in motion behind her back. The child was uader observation for only about three months, as her parents, being very poor, had to leave the neighbourhood to obtain work; but before going away she had learned to recognise her own name, and responded readily when it was uttered. Up to the time of my losing sight of the case I had never been able to inflate the right drum, but probably should have done so later on, especially if I had used the tympanic catheter. It was very noticeable that on the occurrence of any sudden sound the child invariably turned towards the left, the hearing side. The only other case of deaf-mutism that has been brought to me for treatment was certainly congenital, and curative treatment was impractlcable.

Twenty years or more ago a French surgeon, whose name I forget, suggested that deaf-mutism might bo due to Eustachian obstruction leading to retraction of the membrana bympani and consequent pressure upon or strebching of the chorda tympani, and that the injury to this nerve was the cause of dumbness, from which, perhaps, one may infer that he regarded the chorda tympani as a motor nerve of the tongue, or as being in some way concerned in the production of articulate speech, whereas it is a secretory nerve as to the submaxillary and sublingual glands and sensory as to the tongue. Any cause of total deafness in an infant would prevent speech, and therefore in all cases of deaf.mutism in children a removable source of deafness should be carefully sought and, if found, suitably treated. In connexion with this subject I wonld call particular attention to the use of the tympanic catheter, by means of which the drum may sometimes be successfully inflated when all other means have failed. I have also used it with excellent results to wash out an accumulation of mucus from the tympanic cavity, employing for that purpose a warm solntion of pure carbonate of soda, four grains to the ounce. Gloucester-place, W.

\section{Clinital a}

\section{MEDICAL, SURGICAL, OBSTETRICAL, AND THERAPEUTICAL.}

\section{AN ANEURYSM OF THE SUPRA-ORBITAL ARTERY CURED BY PRESSURE APPLIED BY THE} PATIENT'S THUMB.

BY WM. MURRAY, M.D., F.R.C.P. LoND.

The case of "Cirsoid Aneurysm of the Eyebrow" shown lately by Mr. G. R. Turner at the meebing of the Clinical Society recalls a case which seems worthy of record. The subject of this aneurysm was captain of a ship, and was treated in Charlestown, U.S , first of all by digital pressure and then by proximal ligature, for a small aneurysm of the supra-orbital arbery, about three-quarters of an inch above the notch. Although the sac seemed to be consoli. dabed and partly absorbed before he left Charlestown, another pulsating swelling appeared during his nexb long voyage. Having had experience of the digital preseure treatment, he of his own accord, while at sea, applied pressure in the following manner with success. He sat on chair in front of a strong table and placed the right elbow on his knee, the uplifted forearm being supported by the edge of the table in front of him. The head was then brought down on the thumb, which was applied on the neas side of the aneurysm just above the supra orbital notch wibh sufficient firmness to stop pulsation in the $s$ welling above. In this position the poor fellow, supported by the sympathy and succour of his men, held on for several hours, and was rewarded by finding that the tumour had becoma quite pulseless and solid. A few months after this 1 